6. S. Banach and S. Mazur, Uber mehrdeutige stetige Abbildungen, Studia Math. vol. 6 (1936) pp. 174-178.

7. Ernest Michael, Topologies on spaces of subsets, Trans. Amer. Math. Soc. vol. 71 (1951) pp. 152-182.

8. P. Alexandroff and H. Hopf, Topologie, Berlin, 1935. 283.

9. J. L. Kelley, Convergence in topology, Duke Math. J. vol. 17 (1950) pp. 277-

University of Miami

\title{
ERRATA, VOLUME 3
}

C. W. Curtis, $A$ note on noncommutative polynomials.

p. 965, line 10 from the bottom. Add to condition (b): "where $T(r) \neq 0$ if $r \neq 0 . "$

\section{ERRATA, VOLUME 4}

W. R. Mann, Mean value methods in iteration.

p. 507, Display (2) should include the following:

$$
\lim _{i \rightarrow \infty} a_{i j}=0 \quad \text { for all } j \text {. }
$$

E. Michael, $A$ note on paracompact spaces.

p. 835, diagram near the top of the page. For "covering" read "open covering" (twice), and for "refinement" read "open refinement" (twice). 